\title{
OPERATOR IDEALS ARISING FROM GENERATING SEQUENCES
}

\author{
NGAI-CHING WONG \\ Dedicated to Professor Kar-Ping Shum in honor of his seventieth birthday
}

\begin{abstract}
In this note, we will discuss how to relate an operator ideal on Banach spaces to the sequential structures it defines. Concrete examples of ideals of compact, weakly compact, completely continuous, Banach-Saks and weakly Banach-Saks operators will be demonstrated.
\end{abstract}

\section{INTRODUCTION}

Let $T: E \rightarrow F$ be a linear operator between Banach spaces. Let $U_{E}, U_{F}$ be the closed unit balls of $E, F$, respectively. Note that closed unit balls serve simultaneously the basic model for open sets and bounded sets in Banach spaces. The usual way to describe $T$ is to state either the bornological property, via $T U_{E}$, or the topological property, via $T^{-1} U_{F}$, of $T$. An other way classifying $T$ is through the sequential structures $T$ preserves. Here are some well-known examples.

Examples 1.1. (1) $\quad T$ is bounded (i.e., $T U_{E}$ is a bounded subset of $F$ ) $\Leftrightarrow T$ is continuous (i.e., $T^{-1} U_{F}$ is a 0 -neighborhood of $E$ in the norm topology) $\Leftrightarrow T$ is sequentially bounded ( i.e., $T$ sends bounded sequences to bounded sequences);

(2) $T$ is of finite rank (i.e., $T U_{E}$ spans a finite dimensional subspace of $F$ ) $\Leftrightarrow T$ is weak-norm continuous (i.e., $T^{-1} U_{F}$ is a 0 -neighborhood of $E$ in the weak topology)

$\Leftrightarrow T$ sends bounded sequences to sequences spanning finite dimensional subspaces of $F$;

Date: January 11, 2011; for the Proceedings of ICA 2010 (World Scientific).

2000 Mathematics Subject Classification. 47L20, 47B10 46A11, 46A17.

Key words and phrases. operator ideals, generating sequences, generating bornologies.

Partially supported by a Taiwan National Science Council grant 99-2115-M-110-007-MY3. 
$T$ is compact (i.e., $T U_{E}$ is totally bounded in $F$ )

$\Leftrightarrow T$ is continuous in the topology of uniform convergence on norm compact subsets of $E^{\prime}$ (i.e., $T^{-1} U_{F} \supseteq K^{\circ}$, the polar of a norm compact subset $K$ of the dual space $E^{\prime}$ of $E$ )

$\Leftrightarrow T$ is sequentially compact (i.e., $T$ sends bounded sequences to sequences with norm convergent subsequences); and

(4) $T$ is weakly compact (i.e., $T U_{E}$ is relatively weakly compact in $F$ )

$\Leftrightarrow T$ is continuous in the topology of uniform convergence on weakly compact subsets of $E^{\prime}$ (i.e., $T^{-1} U_{F} \supseteq K^{\circ}$, the polar of a weakly compact subset $K$ of $E^{\prime}$ ) $\Leftrightarrow T$ is sequentially weakly compact (i.e., $T$ sends bounded sequences to sequences with weakly convergent subsequences).

In [18, 17, 15], we investigate the duality of the topological and bornological characters of operators demonstrated in the above examples, and applied them in the study of operator ideal theory in the sense of Pietsch [6]. In [16], we use these concepts in classifying locally convex spaces. They are also used by other authors in operator algebra theory in [13, 2].

After giving a brief account of the equivalence among the notions of operator ideals, generating topologies, and generating bornologies developed in [18, 17, 15] in Section 2, we shall explore into the other option of using sequential structures in Section 3. The theory is initialed by Stephani [11, 12]. We provide a variance here. In particular, we will show that the notions of operator ideals, generating bornologies and generating sequences are equivalent in the context of Banach spaces. However, we note that the sequential methodology does not seem to be appropriate in the context of locally convex spaces, as successfully as in [18, 16]. Some examples of popular operator ideals are treated in our new ways as demonstrations in Section 4.

The author would like to take this opportunity to thank Professor Kar-Ping Shum. He has learned a lot from Professor Shum since he was a student in the Chinese University of Hong Kong in 1980's. With about 300 publications, Professor Shum has served as a good model for the author and other fellows since then. The current work is based partially on the thesis of the author finished when he was studying with Professor Shum. 


\section{The triangle of operators, topologies And BORnOlogies}

Let $X$ be a vector space over $\mathbb{K}=\mathbb{R}$ or $\mathbb{C}$. Following Hogbe-Nlend [4], by a vector bornology on $X$ we mean a family $\mathcal{B}$ of subsets of $X$ satisfies the following conditions:

$\left(\mathrm{VB}_{1}\right) X=\cup \mathcal{B}$

$\left(\mathrm{VB}_{2}\right)$ if $B \in \mathcal{B}$ and $A \subseteq B$ then $A \in \mathcal{B}$;

$\left(\mathrm{VB}_{3}\right) B_{1}+B_{2} \in \mathcal{B}$ whenever $B_{1}, B_{2} \in \mathcal{B}$;

$\left(\mathrm{VB}_{4}\right) \lambda B \in \mathcal{B}$ whenever $\lambda \in \mathbb{K}$ and $B \in \mathcal{B}$;

$\left(\mathrm{VB}_{5}\right)$ the circle hull $\mathrm{ch} B$ of any $B$ in $\mathcal{B}$ belongs to $\mathcal{B}$.

Elements in $\mathcal{B}$ are called $\mathcal{B}$-bounded sets in $X$. A vector bornology $\mathcal{B}$ on $X$ is called a convex bornology if $\Gamma B \in \mathcal{B}$ for all $B$ in $\mathcal{B}$, where $\Gamma B$ is the absolutely convex hull of $B$. The pair $(X, \mathcal{B})$ is called a convex bornological space which is denoted by $X^{\mathcal{B}}$.

Let $(X, \mathcal{T})$ be a locally convex space. For any subset $B$ of $X$, the $\sigma$-disked hull of $B$ is defined to be

$$
\Gamma_{\sigma} B=\left\{\sum_{n=1}^{\infty} \lambda_{n} b_{n}: \sum_{n=1}^{\infty}\left|\lambda_{n}\right| \leq 1, \lambda_{n} \in \mathbb{K}, b_{n} \in B, n=1,2, \ldots\right\}
$$

A set $B$ in $X$ is said to be $\sigma$-disked if $B=\Gamma_{\sigma} B$. An absolutely convex, bounded subset $B$ of $X$ is said to be infracomplete (or a Banach disk) if the normed space $\left(X(B), r_{B}\right)$ is complete, where

$$
X(B)=\bigcup_{n \geq 1} n B \quad \text { and } \quad r_{B}(x):=\inf \{\lambda>0: x \in \lambda B\}
$$

is the gauge of $B$ defined on $X(B)$.

Lemma $2.1([18])$. Let $(X, \mathcal{P})$ be a locally convex space and $B$ an absolutely convex bounded subset of $X$.

(a) If $B$ is $\sigma$-disked then $B$ is infracomplete.

(b) If $B$ is infracomplete and closed then $B$ is $\sigma$-disked.

Let $X, Y$ be locally convex spaces. Denote by $\sigma\left(X, X^{\prime}\right)$ the weak topology of $X$ with respect to its dual space $X^{\prime}$, while $\mathcal{P}_{\text {ori }}(X)$ is the original topology of $X$. We employ the notion $\mathcal{M}_{\text {fin }}(Y)$ for the finite dimensional bornology of $Y$ which has a basis consisting of 
all convex hulls of finite sets. On the other hand, $\mathcal{M}_{\mathrm{von}}(Y)$ is used for the von Neumann bornology of $Y$ which consists of all $\mathcal{P}_{\text {ori }}(Y)$-bounded subsets of $Y$. Ordering of topologies and bornologies are induced by set-theoretical inclusion, as usual. Moreover, we write briefly $X_{\mathcal{P}}$ for a vector space $X$ equipped with a locally convex topology $\mathcal{P}$ and $Y^{\mathcal{M}}$ for a vector space $Y$ equipped with a convex vector bornology $\mathcal{M}$.

Let $X, Y$ be locally convex spaces. We denote by $\mathfrak{L}(X, Y)$ and $L^{\times}(X, Y)$ the collection of all linear operators from $X$ into $Y$ which are continuous and (locally) bounded (i.e., sending bounded sets to bounded sets), respectively.

Definition 2.2 (see [16]). Let $\mathcal{C}$ be a subcategory of locally convex spaces.

(1) ("Operators") A family $\mathfrak{A}=\{\mathfrak{A}(X, Y): X, Y \in \mathcal{C}\}$ of algebras of operators associated to each pair of spaces $X$ and $Y$ in $\mathcal{C}$ is called an operator ideal if

$\mathrm{OI}_{1}: \mathfrak{A}(X, Y)$ is a nonzero vector subspace of $\mathfrak{L}(X, Y)$ for all $X, Y$ in $\mathcal{C}$; and $\mathrm{OI}_{2}: R T S \in \mathfrak{A}\left(X_{0}, Y_{0}\right)$ whenever $R \in \mathfrak{L}\left(Y, Y_{0}\right), T \in \mathfrak{A}(X, Y)$ and $S \in$ $\mathfrak{L}\left(X_{0}, X\right)$ for any $X_{0}, X, Y$ and $Y_{0}$ in $\mathrm{e}$.

(2) ("Topologies") A family $\mathcal{P}=\{\mathcal{P}(X): X \in \mathcal{C}\}$ of locally convex topologies associated to each space $X$ in $\mathcal{C}$ is called a generating topology if

$\mathrm{GT}_{1}: \sigma\left(X, X^{\prime}\right) \subseteq \mathcal{P}(X) \subseteq \mathcal{P}_{\text {ori }}(X)$ for all $X$ in $\mathcal{C}$; and $\mathbf{G T}_{2}: \mathfrak{L}(X, Y) \subseteq \mathfrak{L}\left(X_{\mathcal{P}}, Y_{\mathcal{P}}\right)$ for all $X$ and $Y$ in $\mathcal{C}$.

(3) ("Bornologies") A family $\mathcal{M}=\{\mathcal{N}(Y): Y \in \mathcal{C}\}$ of convex vector bornologies associated to each space $Y$ in $\mathcal{C}$ is called a generating bornology if

$\mathbf{G B}_{1}: \mathcal{M}_{\text {fin }}(Y) \subseteq \mathcal{M}(Y) \subseteq \mathcal{M}_{\text {von }}(Y)$ for all $Y$ in $\mathcal{C}$; and $\mathbf{G B}_{2}: \mathfrak{L}(X, Y) \subseteq L^{\times}\left(X^{\mathcal{M}}, Y^{\mathcal{M}}\right)$ for all $X$ and $Y$ in $\mathcal{C}$.

Definition 2.3. Let $\mathfrak{A}$ be an operator ideal, $\mathcal{P}$ a generating topology and $\mathcal{M}$ a generating bornology on $\mathrm{C}$.

(1) ("Operators" $\rightarrow$ "Topologies") For each $X_{0}$ in $\mathcal{C}$, the $\mathfrak{A}$-topology of $X_{0}$, denoted by $\mathcal{T}(\mathfrak{A})\left(X_{0}\right)$, is the projective topology of $X_{0}$ with respect to the family

$$
\left\{T \in \mathfrak{A}\left(X_{0}, Y\right): Y \in \mathcal{C}\right\}
$$


In other words, a seminorm $p$ of $X_{0}$ is $\mathcal{T}(\mathfrak{A})\left(X_{0}\right)$-continuous if and only if there is a $T$ in $\mathfrak{A}\left(X_{0}, Y\right)$ for some $Y$ in $\mathcal{C}$ and a continuous seminorm $q$ of $Y$ such that

$$
p(x) \leq q(T x), \quad \forall x \in X_{0}
$$

In this case, we call $p$ an $\mathfrak{A}$-seminorm of $X_{0}$.

(2) ("Operators" $\rightarrow$ "Bornologies") For each $Y_{0}$ in $\mathcal{C}$, the $\mathfrak{A}$-bornology of $Y_{0}$, denoted by $\mathcal{B}(\mathfrak{A})\left(Y_{0}\right)$, is the inductive bornology of $Y_{0}$ with respect to the family

$$
\left\{T \in \mathfrak{A}\left(X, Y_{0}\right): X \in \mathcal{C}\right\} .
$$

In other words, a subset $B$ of $Y_{0}$ is $\mathcal{B}(\mathfrak{A})\left(Y_{0}\right)$-bounded if and only if there is a $T$ in $\mathfrak{A}\left(X, Y_{0}\right)$ for some $X$ in $\mathcal{C}$ and a topologically bounded subset $A$ of $X$ such that

$$
B \subseteq T A
$$

In this case, we call $B$ an $\mathfrak{A}$-bounded subset of $Y_{0}$.

(3) ("Topologies" $\rightarrow$ "Operators") For $X, Y$ in $\mathcal{C}$, let

$$
\mathcal{O}(\mathcal{P})(X, Y)=\mathfrak{L}\left(X_{\mathcal{P}}, Y\right)
$$

be the vector space of all continuous operators from $X$ into $Y$ which is still continuous with respect to the $\mathcal{P}(X)$-topology.

(4) ("Bornologies" $\rightarrow$ "Operators") For $X, Y$ in $\mathcal{C}$, let

$$
\mathcal{O}(\mathcal{M})(X, Y)=\mathfrak{L}(X, Y) \cap L^{\times}\left(X, Y^{\mathcal{M}}\right)
$$

be the vector space of all continuous operators from $X$ into $Y$ which send bounded sets to $\mathcal{M}(Y)$-bounded sets.

(5) ("Topologies" $\leftrightarrow$ "Bornologies") For $X, Y$ in $\mathcal{C}$, the $\mathcal{P}^{\circ}(Y)$-bornology of $Y$ (resp. $\mathcal{M}^{\circ}(X)$-topology of $X$ ) is defined to be the bornology (resp. topology) polar to $\mathcal{P}(X)$ (resp. $\mathcal{M}(Y)$ ). More precisely,

- a bounded subset $A$ of $Y$ is $\mathcal{P}^{\circ}(Y)$-bounded if and only if its polar $A^{\circ}$ is a $\mathcal{P}\left(Y_{\beta}^{\prime}\right)$-neighborhood of zero; and

- a neighborhood $V$ of zero of $X$ is a $\mathcal{M}^{\circ}(X)$-neighborhood of zero if and only if $V^{\circ}$ is $\mathcal{M}\left(X_{\beta}^{\prime}\right)$-bounded.

Here are two examples: the ideals $\mathfrak{K}$ of compact operators and $\mathfrak{P}$ of absolutely summing operators (see e.g. [6]), the generating systems $\mathcal{P}_{p c}$ of precompact topologies (see 
e.g. [7]) and $\mathcal{P}_{p n}$ of prenuclear topologies (see e.g. [8, p. 90]), and the generating systems $\mathcal{M}_{p c}$ of precompact bornologies and $\mathcal{M}_{p n}$ of prenuclear bornologies (see e.g. [5]), respectively.

Definition 2.4. An operator ideal $\mathfrak{A}$ on Banach spaces is said to be

(1) injective if $S \in \mathfrak{A}\left(E, F_{0}\right)$ infers $T \in \mathfrak{A}(E, F)$, whenever $T \in \mathfrak{L}(E, F)$ and $\|T x\| \leq$ $\|S x\|, \forall x \in E$;

(2) surjective if $S \in \mathfrak{A}\left(E_{0}, F\right)$ infers $T \in \mathfrak{A}(E, F)$, whenever $T \in \mathfrak{L}(E, F)$ and $T U_{E} \subseteq S U_{E_{0}}$.

The injective hull $\mathfrak{A}^{\text {inj }}$ and the surjective hull $\mathfrak{A}^{\text {sur }}$, of $\mathfrak{A}$ is the intersection of all injective and surjective operator ideals containing $\mathfrak{A}$, respectively. For example, $\mathfrak{K}=$ $\mathfrak{K}^{\text {inj }}=\mathfrak{K}^{\text {sur }}$ and $\mathfrak{P}=\mathfrak{P}^{\text {inj }} \varsubsetneqq \mathfrak{P}^{\text {sur }}$ (see, e.g., [6]).

In the following, we see that the notions of operator ideals, generating topologies, and generating bornologies are equivalent (see also [13] for the operator algebra version).

Theorem $2.5([9,10,18,16])$. Let $\mathfrak{A}$ be an operator ideal, $\mathcal{P}$ a generating topology and $\mathcal{M}$ a generating bornology on Banach spaces. We have

(1) $\mathcal{T}(\mathfrak{A})=\{\mathcal{T}(\mathfrak{A})(X): X \in \mathcal{C}\}$ is a generating topology.

(2) $\mathcal{B}(\mathfrak{A})=\{\mathcal{B}(\mathfrak{A})(Y): Y \in \mathcal{C}\}$ is a generating bornology.

(3) $\mathcal{O}(\mathcal{P})=\{\mathcal{O}(\mathcal{P})(X, Y): X, Y \in \mathcal{C}\}$ is an operator ideal.

(4) $\mathcal{O}(\mathcal{M})=\{\mathcal{O}(\mathcal{M})(X, Y): X, Y \in \mathcal{C}\}$ is an operator ideal.

(5) $\mathcal{P}^{\circ}=\left\{\mathcal{P}^{\circ}(Y): Y \in \mathcal{C}\right\}$ is a generating bornology.

(6) $\mathcal{M}^{\circ}=\left\{\mathcal{M}^{\circ}(Y): Y \in \mathcal{C}\right\}$ is a generating topology.

(7) $\mathcal{O}(\mathfrak{T}(\mathfrak{A}))=\mathfrak{A}^{\mathrm{inj}}$.

(8) $\mathcal{O}(\mathcal{B}(\mathfrak{A}))=\mathfrak{A}^{\text {sur }}$.

(9) $\mathcal{T}(\mathcal{O}(\mathcal{P}))=\mathcal{P}$.

(10) $\mathcal{B}(\mathcal{O}(\mathcal{M}))=\mathcal{M}$. 
For generating topologies $\mathcal{P}$ and $\mathcal{P}_{1}$, and generating bornologies $\mathcal{M}$ and $\mathcal{M}_{1}$ on Banach spaces, we can also associate to them the operator ideals with components

$$
\begin{aligned}
\mathcal{O}\left(\mathcal{P} / \mathcal{P}_{1}\right)(X, Y) & =\mathfrak{L}\left(X_{\mathcal{P}}, Y_{\mathcal{P}_{1}}\right), \\
\mathcal{O}\left(\mathcal{M} / \mathcal{M}_{1}\right)(X, Y) & =L^{\times}\left(X^{\mathcal{M}_{1}}, Y^{\mathcal{M}}\right) \cap \mathfrak{L}(X, Y) \\
\mathcal{O}(\mathcal{P} / \mathcal{M})(X, Y) & =\mathfrak{L}\left(X_{\mathcal{P}}, Y^{\mathcal{M}}\right) .
\end{aligned}
$$

These give rise to

$$
\begin{aligned}
\mathcal{O}\left(\mathcal{P} / \mathcal{P}_{1}\right) & =\mathcal{O}\left(\mathcal{P}_{1}\right)^{-1} \circ \mathcal{O}(\mathcal{P}) \\
\mathcal{O}\left(\mathcal{M} / \mathcal{M}_{1}\right) & =\mathcal{O}(\mathcal{M}) \circ \mathcal{O}\left(\mathcal{M}_{1}\right)^{-1} \quad[12] \\
\mathcal{O}(\mathcal{P} / \mathcal{M}) & =\mathcal{O}(\mathcal{M}) \circ \mathcal{O}(\mathcal{P})=\mathcal{O}(\mathcal{M}) \circ \mathcal{O}(\mathcal{P})
\end{aligned}
$$

Here, the product $\mathfrak{A} \circ \mathfrak{B}$ consists of operators of the form $S T$ with $S \in \mathfrak{A}$ and $T \in \mathfrak{B}$, while the quotient $\mathfrak{A} \circ \mathfrak{B}^{-1}$ consists of those $S$ such that $S T \in \mathfrak{A}$ whenever $T \in \mathfrak{B}$. Readers are referred to Pietsch's classic [6] for information regarding quotients and products of operator ideals.

\section{Generating Sequences}

The following is based on a version in [11].

Definition 3.1. A family $\Phi(E)$ of bounded sequences in a Banach space $E$ is called a vector sequential structure if

- $\left\{a x_{n}+b y_{n}\right\} \in \Phi(E)$ whenever $\left\{x_{n}\right\},\left\{y_{n}\right\} \in \Phi(E)$ and $a, b$ are scalars;

- every subsequence of a sequence in $\Phi(E)$ belongs to $\Phi(E)$.

Clearly, the biggest vector sequential structure on a Banach space $E$ is the family $\Phi_{b}(E)$ of all bounded sequences in $E$. On the other hand, we let $\Phi_{\text {fin }}(E)$ to be the vector sequential structure on $E$ of all bounded sequences $\left\{x_{n}\right\}$ with finite dimensional ranges, i.e., there is a finite dimensional subspace of $F$ containing all $x_{n}$ 's.

Given two Banach spaces $E, F$ with vector sequential structures $\Phi, \Psi$, we let $\mathfrak{L}\left(E^{\Phi}, F^{\Psi}\right)$ be the vector space of all continuous linear operators sending a bounded sequence in $\Phi$ to a bounded sequence in $F$ with a subsequence in $\Psi$. 
Definition 3.2. A family $\Phi:=\{\Phi(F): F$ is a Banach space $\}$ of (bounded) vector sequential structures on each Banach space is called a generating sequential structure if

$\mathbf{G S}_{1}: \Phi_{\text {fin }}(F) \subseteq \Phi(F) \subseteq \Phi_{b}(F)$ for every Banach space $F$.

$\mathbf{G S}_{2}: \mathfrak{L}(E, F) \subseteq \mathfrak{L}\left(E^{\Phi}, F^{\Phi}\right)$ for all Banach spaces $E, F$.

Definition 3.3. Let $\mathfrak{A}$ be an operator ideal on Banach spaces. Call a bounded sequence $\left\{y_{n}\right\}$ in a Banach space $F$ an $\mathfrak{A}$-sequence if there is a bounded sequence $\left\{x_{n}\right\}$ in a Banach space $E$ and a continuous linear operator $T$ in $\mathfrak{A}(E, F)$ such that $T x_{n}=y_{n}, n=1,2, \ldots$. Denote by $\mathcal{S}(\mathfrak{A})(F)$ the family of all $\mathfrak{A}$-sequences in $F$.

Lemma 3.4. Let $\mathfrak{A}$ be an operator ideal on Banach spaces. Then

$$
\mathcal{S}(\mathfrak{A}):=\{\mathcal{S}(\mathfrak{A})(F): F \text { is a Banach space }\}
$$

is a generating sequential structure.

Proof. It is easy to see that all $\mathcal{S}(\mathfrak{A})(F)$ is a bounded vector sequential structure. Since the ideal $\mathfrak{F}$ of continuous linear operators of finite rank is the smallest operator ideal, while the ideal $\mathfrak{L}$ of all continuous linear operators is the biggest, we see that $\left(\mathrm{GS}_{1}\right)$ is satisfied. On the other hand, $\left(\mathrm{GS}_{2}\right)$ follows from $\left(\mathrm{OI}_{2}\right)$ directly.

We now work on the converse of Lemma 3.4. Let $\Phi$ be a generating sequential structure. Denote by $\mathcal{M}_{\text {base }}^{\Phi}(E)$ the family of all bounded sets $M$ in a Banach space $E$ such that every sequence $\left\{x_{n}\right\}$ in $M$ has a subsequence $\left\{x_{n_{k}}\right\}$ in $\Phi(E)$.

Lemma 3.5. The family $\mathcal{M}^{\Phi}(E)$ of all (bounded) sets with circle hull in $\mathcal{M}_{\text {base }}^{\Phi}(E)$ in a Banach space $E$ forms a generating bornology $\mathcal{M}^{\Phi}$.

Proof. Let $M, N \in \mathcal{M}^{\Phi}(E)$, let $\lambda$ be a scalar, and let $W \subseteq M$. It is easy to see that the circle hull ch $M, \lambda M$ and $W$ are all in $\mathcal{M}^{\Phi}(E)$. For the sum $M+N$, we first notice that both ch $M$ and ch $N$ belong to $\mathcal{M}_{\text {base }}^{\Phi}(E)$. Now

$$
M+N \subseteq \operatorname{ch} M+\operatorname{ch} N \in \mathcal{M}_{\text {base }}^{\Phi}(E)
$$

and

$$
\operatorname{ch}(M+N) \subseteq \operatorname{ch} M+\operatorname{ch} N
$$


give $M+N \in \mathcal{M}^{\Phi}(E)$. All these together say that $\mathcal{M}^{\Phi}(E)$ is a vector bornology on the Banach space $E$.

The condition $\left(\mathrm{GB}_{1}\right)$ follows directly from $\left(\mathrm{GS}_{1}\right)$. For $\left(\mathrm{GB}_{2}\right)$, let $T \in \mathfrak{L}(E, F)$ and $M \in \mathcal{M}^{\Phi}(E)$. Then for every sequence $\left\{T x_{n}\right\}$ in $T M$, we will have a subsequence of $\left\{x_{n}\right\}$ in $\Phi(E)$. By $\left(\mathrm{GS}_{2}\right)$, we have a further subsequence $\left\{T x_{n_{k}}\right\}$ belonging to $\mathcal{M}^{\Phi}(F)$.

Definition 3.6. A generating sequential structure $\Phi$ is said to be normal if for every $\left\{x_{n}\right\}$ in $\Phi(E)$ and every bounded scalar sequence $\left(\lambda_{n}\right)$ in $\ell^{\infty}$ of norm not greater than 1, we have $\left\{\lambda_{n} x_{n}\right\} \in \Phi(E)$.

It is clear that $\mathcal{S}(\mathfrak{A})$ is normal for any operator ideal $\mathfrak{A}$.

Lemma 3.7. For every normal generating sequential structure $\Phi$, we have

$$
\mathcal{M}_{\text {base }}^{\Phi}=\mathcal{M}^{\Phi}
$$

Proof. It suffices to show that every $M$ in $\mathcal{M}_{\text {base }}^{\Phi}(E)$ has its circle hull ch $M$ in $\mathcal{M}_{\text {base }}^{\Phi}(E)$ for each Banach space $E$, i.e., $\mathcal{M}_{\text {base }}^{\Phi}$ is itself circled. To this end, let $\left\{x_{n}\right\}$ be from $\operatorname{ch} M$. Then there is a $\left(\lambda_{n}\right)$ from $\ell^{\infty}$ of norm not greater than 1 such that

$$
x_{n}=\lambda_{n} y_{n}, \quad n=1,2, \ldots
$$

for some $\left\{y_{n}\right\}$ in $M$. Now, there is a subsequence $\left\{y_{n_{k}}\right\}$ in $\Phi(E)$ ensuring that $\left\{x_{n_{k}}\right\}=$ $\left\{\lambda_{n_{k}} y_{n_{k}}\right\} \in \Phi(E)$ for $\Phi$ being normal.

Definition 3.8. Let $\Phi$ be a generating sequential structure on Banach spaces. Let $E, F$ be Banach spaces. Denote by $\mathcal{O}(\Phi)(E, F)$ the family of all continuous linear operators $T$ in $\mathfrak{L}(E, F)$ sending every bounded sequence $\left\{x_{n}\right\}$ in $E$ to a bounded sequence in $F$ with a subsequence $\left\{T x_{n}\right\}$ in $\Phi(F)$. In other words, $\mathcal{O}(\Phi)(E, F)=\mathfrak{L}\left(E^{\Phi_{b}}, F^{\Phi}\right)$.

Theorem 3.9. Let $\Phi$ be a generating sequential structure on Banach spaces. Then $\mathcal{O}(\Phi)$ is a surjective operator ideal. More precisely,

$$
\mathcal{O}(\Phi)=\mathcal{O}\left(\mathcal{M}^{\Phi}\right)
$$

Proof. The ideal property of $\mathcal{O}(\Phi)$ is trivial. For the surjectivity of $\mathcal{O}(\Phi)$, we let $S \in$ $\mathcal{O}(\Phi)\left(E_{0}, F\right)$ and $T \in \mathfrak{L}(E, F)$ such that $T U_{E} \subseteq S U_{E_{0}}$. Here, $U_{E}, U_{E_{0}}$ are the closed 
unit balls of $E, E_{0}$, respectively. We need to show that $T \in \mathcal{O}(\Phi)(E, F)$. In fact, for any bounded sequence $\left\{x_{n}\right\}$ in $E$, we have

$$
T x_{n}=S y_{n}, \quad n=1,2, \ldots,
$$

with some bounded sequence $\left\{y_{n}\right\}$ in $F$. Now, the fact $\left\{S y_{n}\right\}$ has a subsequence $\left\{S y_{n_{k}}\right\} \in$ $\Phi(F)$ implies that $T \in \mathcal{O}(\Phi)(E, F)$, as asserted.

Finally, we check the representation. The inclusion $\mathcal{O}(\Phi) \subseteq \mathcal{O}\left(\mathcal{M}^{\Phi}\right)$ is an immediate consequence of the definition of $\mathcal{M}^{\Phi}$. Conversely, let $T \in \mathcal{O}\left(\mathcal{M}^{\Phi}\right)(E, F)$, i.e., $T U_{E} \in$ $\mathcal{M}^{\Phi}(F)$. Let $\left\{x_{n}\right\}$ be a bounded sequence in $E$. We can assume $\left\|x_{n}\right\| \leq 1$ for all $n$. Then $\left\{T x_{n}\right\} \subseteq T U_{E}$ and hence possesses a subsequence $\left\{T x_{n_{k}}\right\} \in \Phi(F)$. In other words, $T \in \mathcal{O}(\Phi)(E, F)$.

Corollary 3.10. If a generating sequential structure $\Phi$ is normal then

$$
\mathcal{S}(\mathcal{O}(\Phi))=\Phi
$$

Conversely, if $\mathfrak{A}$ is an operator ideal on Banach spaces then

$$
\mathcal{O}(\mathcal{S}(\mathfrak{A}))=\mathfrak{A}^{\text {sur }}
$$

Theorem 3.11. Let $\Phi, \Psi$ be two generating sequential structures. Let

$$
\mathcal{O}(\Phi / \Psi)(E, F):=\mathfrak{L}\left(E^{\Psi}, F^{\Phi}\right)
$$

i.e., $T \in \mathcal{O}(\Phi / \Psi)(E, F)$ if and only if the continuous linear operator $T$ sends each bounded sequence $\left\{x_{n}\right\}$ in $\Psi(E)$ to a sequence in $F$ with a subsequence $\left\{T x_{n_{k}}\right\}$ in $\Phi(F)$. Then $\mathcal{O}(\Phi / \Psi)(E, F)$ is an operator ideal on Banach spaces. Moreover, if $\Psi$ is normal, then

$$
\mathcal{O}(\Phi / \Psi)=\mathcal{O}\left(\mathcal{M}^{\Phi} / \mathcal{M}^{\Psi}\right)=\mathcal{O}\left(\mathcal{M}^{\Phi}\right) \circ \mathcal{O}\left(\mathcal{M}^{\Psi}\right)^{-1}
$$

Proof. Conditions $\left(\mathrm{OI}_{1}\right)$ and $\left(\mathrm{OI}_{2}\right)$ follow from Conditions $\left(\mathrm{GS}_{1}\right)$ and $\left(\mathrm{GS}_{2}\right)$, respectively.

Assume $\Psi$ is normal. Let $T \in \mathcal{O}(\Phi / \Psi)(E, F)$ and $M \in \mathcal{M}^{\Psi}(E)$ be circled. Suppose, on contrary, $T M \notin \mathcal{M}^{\Phi}(F)$. By definition, there is a sequence $\left\{T x_{n}\right\}$ in $T M$ having no subsequence $\left\{T x_{n_{k}}\right\}$ in $\Phi(F)$. But since $\left\{x_{n}\right\} \subseteq M$, it has a subsequence $\left\{x_{n_{k}}\right\}$ in $\Psi(E)$. And thus $\left\{T x_{n_{k}}\right\}$ has a further subsequence in $\Phi(F)$, a contradiction. 
Conversely, let $T \in \mathcal{O}\left(\mathcal{M}^{\Phi} / \mathcal{M}^{\Psi}\right)(E, F)$. It is easy to see that the range of any sequence $\left\{x_{n}\right\}$ in $\Psi(E)$ is a bounded set in $\mathcal{M}^{\psi}(E)$, by noting that $\Psi$ is normal. The range of the sequence $\left\{T x_{n}\right\}$ is bounded in $\mathcal{M}^{\Phi}(F)$. Therefore, there is a subsequence $\left\{T x_{n_{k}}\right\}$ of $\left\{T x_{n}\right\}$ belongs to $\Phi(F)$. This ensures $T \in \mathcal{O}(\Phi / \Psi)(E, F)$.

Definition 3.12. Given two generating sequential structures $\Phi$ and $\Psi$. We say that $\Psi(E)$ is coordinated to $\Phi(E)$ if the following conditions holds. Suppose $\left\{x_{n}\right\} \in \Psi(E)$. The bounded sequence $\left\{x_{n}\right\} \notin \Phi(E)$ if and only if $\left\{x_{n}\right\}$ has a subsequence $\left\{x_{n_{k}}\right\}$ which has no further subsequence belonging to $\Phi(E)$. We write, in this case, $\Phi(E) \ll \Psi(E)$. If $\Phi(E) \ll \Psi(E)$ for all Banach spaces $E$ then we write $\Phi \ll \Psi$.

Theorem 3.13. Assume $\Phi, \Psi$ be two generating sequential structures such that $\Phi(F) \ll$ $\Psi(F)$ for some Banach space $F$. Then $\mathcal{O}(\Phi / \Psi)(E, F)$ consists of exactly those continuous linear operators sending bounded sequences in $\Psi(E)$ to bounded sequences in $\Phi(F)$ for any Banach space E.

Proof. Assume $T \in \mathcal{O}(\Phi / \Psi)(E, F)$ and $\left\{x_{n}\right\} \in \Psi(E)$. Now, suppose $\left\{T x_{n}\right\} \notin \Phi(F)$. Since $\left\{T x_{n}\right\} \in \Psi(F)$ by $\left(\mathrm{GS}_{2}\right)$, we can verify the condition in Definition 3.12, Therefore, we might have a subsequence $\left\{T x_{n_{k}}\right\}$ having no further subsequence in $\Phi(F)$. However, by the facts that $\left\{x_{n_{k}}\right\} \in \Psi(E)$ and $T \in \mathcal{O}(\Phi / \Psi)(E, F)$, we arrive at the asserted contradiction.

Corollary 3.14. Let $\Phi, \Psi$ be two generating sequential structures such that $\Phi \ll \Psi$ and $\Psi$ is normal. Then, the operator ideal $\mathcal{O}\left(\mathcal{M}^{\Phi}, \mathcal{M}^{\Psi}\right)$ consists of exactly those operators sending sequences in $\Psi$ to sequences in $\Phi$.

\section{EXAMPLES}

We begin with some useful generating bornologies.

Examples 4.1. A set $W$ in a Banach space $F$ is called

(1) nuclear (see, e.g., [11]) if

$$
W \subseteq\left\{y \in F: y=\sum_{n=1}^{\infty} \lambda_{n} y_{n},\left|\lambda_{n}\right| \leq 1\right\}
$$

for an absolutely summable sequence $\left\{y_{n}\right\}$ in $F$; 
(2) unconditionally summable (see, e.g., [11]) if

$$
W \subseteq\left\{y \in F: y=\sum_{n=1}^{\infty} \lambda_{n} y_{n} \text { in norm, }\left|\lambda_{n}\right| \leq 1\right\}
$$

for an unconditionally summable series $\sum_{n=1}^{\infty} y_{n}$ in $F$;

(3) weakly unconditionally summable (see, e.g., [11]) if

$$
W \subseteq\left\{y \in F: y=\sum_{n=1}^{\infty} \lambda_{n} y_{n} \text { weakly, }\left|\lambda_{n}\right| \leq 1\right\}
$$

for a weakly unconditionally summable series $\sum_{n=1}^{\infty} y_{n}$ in $F$;

(4) limited ([1]) if

$$
\lim _{n \rightarrow \infty} \sup _{a \in W}\left|y_{n}^{\prime}(a)\right|=0
$$

for any $\sigma\left(F^{\prime}, F\right)$-null sequence $\left\{y_{n}^{\prime}\right\}$ in $F^{\prime}$.

The above defines generating bornologies $\mathcal{M}_{\nu}, \mathcal{M}_{u c}, \mathcal{M}_{w u c}$, and $\mathcal{M}_{\text {lim }}$, respectively.

Another way to obtain generating bornologies is through generating sequential structures.

Examples 4.2. A bounded sequence $\left\{x_{n}\right\}$ in a Banach space $F$ is called

(1) $\delta_{0}$-fundamental $([11])$ if

$$
t_{n}=x_{n+1}-x_{n}, \quad n=1,2, \ldots,
$$

forms a weakly unconditionally summable series;

(2) limited ([1]) if

$$
\left\langle x_{n}, x_{n}^{\prime}\right\rangle \rightarrow 0 \text { as } n \rightarrow \infty
$$

for any $\sigma\left(F^{\prime}, F\right)$-null sequence $\left\{x_{n}^{\prime}\right\}$ in $F^{\prime}$;

(3) Banach-Saks if

$$
\lim _{n \rightarrow \infty} \frac{x_{1}+x_{2}+\cdots+x_{n}}{n}=x_{0}
$$

for some $x_{0}$ in $F$ in norm.

Examples 4.3. The following are all generating sequential structures.

(1) $\Phi_{\delta_{0}}$ of all $\delta_{0}$-fundamental sequences.

(2) $\Phi_{\lim }$ of all limited sequences.

(3) $\Phi_{\mathrm{BS}}$ of all Banach-Saks sequences. 
(4) $\Phi_{c}$ of all convergent sequences.

(5) $\Phi_{w c}$ of all weakly convergence sequences.

(6) $\Phi_{w C a}$ of all weakly Cauchy sequences.

Examples 4.4 ([11]). The following generating bornologies can be induced by the corresponding generating sequential structures as in Lemma 3.5,

(1) The $\delta_{0}$-compact bornology $\mathcal{M}_{\delta_{0}}$ is defined by $\Phi_{\delta_{0}}$.

(2) The compact bornology $\mathcal{M}_{c}$ is defined by $\Phi_{c}$.

(3) The weakly compact bornology $\mathcal{M}_{w c}$ is defined by $\Phi_{w c}$.

(4) The Rosenthal compact bornology $\mathcal{M}_{w C a}$ is defined by $\Phi_{w C a}$.

(5) The Banach-Saks bornology $\mathcal{M}_{\mathrm{BS}}$ is defined by $\Phi_{\mathrm{BS}}$.

Proposition 4.5. The generating bornology induced by $\Phi_{\lim }$ is $\mathcal{M}_{\lim }$.

Proof. First, we notice that $\Phi_{\lim }$ is normal. Hence, $\mathcal{M}_{\text {base }}^{\Phi_{\text {lim }}}=\mathcal{M}^{\Phi_{\lim }}$ by Lemma 3.7. Let $W \in \mathcal{M}_{\lim }(F)$. Then, by definition,

$$
\lim _{n \rightarrow \infty} \sup _{a \in W}\left|\left\langle a, y_{n}^{\prime}\right\rangle\right|=0
$$

for any $\sigma\left(F^{\prime}, F\right)$-null sequence $\left\{y_{n}^{\prime}\right\}$ in $F^{\prime}$ and any sequence $\left\{y_{n}\right\}$ in $W$. Hence, $W \in$ $\mathcal{M}^{\Phi_{\lim }}(F)$.

Conversely, if $W$ is a bounded set in $F$ such that every sequence $\left\{y_{n}\right\}$ in $W$ has a limited subsequence $\left\{y_{n_{k}}\right\}$, we need to check that $W \in \mathcal{M}_{\text {lim }}$. Assume, on contrary, that there were some $\sigma\left(F^{\prime}, F\right)$-null sequence $\left\{y_{n}^{\prime}\right\}$ in $F^{\prime}$ and some sequence $\left\{a_{n}\right\}$ in $W$ such that

$$
\lim _{n \rightarrow \infty}\left|\left\langle a_{n_{k}}, y_{n_{k}}^{\prime}\right\rangle\right|=0
$$

This is a contradiction and thus $W$ is a member of $\mathcal{M}_{\lim }(F)$.

Proposition 4.6. The generating bornologies $\mathcal{M}_{c}, \mathcal{M}_{w c}, \mathcal{M}_{w C a}, \mathcal{M}_{\nu}, \mathcal{M}_{u c}, \mathcal{M}_{w u c}$ and $\mathcal{M}_{\lim }$ are all $\sigma$-disked.

Proof. For the case of $\mathcal{M}_{\mathrm{lim}}$, see [1]. For all others, see [11].

We are now ready to give a number of examples. 
Examples 4.7. Using Definition 2.3 and Theorem 3.9, we can obtain the following operator ideals on Banach spaces through the associated generating bornologies.

(1) The ideal $\mathfrak{N}^{\text {sur }}$ of co-nuclear operators for the nuclear bornology $\mathcal{M}_{\nu}$.

(2) The ideal $\mathfrak{L}_{\text {lim }}$ of limited operators for the limited bornology $\mathcal{M}_{\text {lim. }}$.

(3) The ideal $\mathfrak{B S}$ of Banach-Saks operators for the Banach-Saks bornology $\mathcal{M}_{\mathrm{BS}}$.

(4) The ideal $\mathfrak{K}$ of compact operators for the compact bornology $\mathcal{M}_{c}$.

(5) The ideal $\mathfrak{W}$ of weakly compact operators for the weakly compact bornology $\mathcal{M}_{w c}$.

(6) The ideal $\mathfrak{R}$ of Rosenthal compact operators for the Rosenthal compact bornology $\mathcal{M}_{w C a}$.

(7) The ideal $\mathfrak{D}_{0}$ of $\delta_{0}$-compact operators for the $\delta_{0}$-compact bornology $\mathcal{M}_{\delta_{0}}$.

For a proof of (a), see [6, p. 112]. For (b), see [3]. For (c), see Theorem 3.9, For others, see [11].

Concerning Theorem 3.13, we have

Examples 4.8. (1) $\Phi_{c} \ll \Phi_{w c}$.

(2) $\Phi_{c} \ll \Phi_{w C a}$.

(3) $\Phi_{w c} \ll \Phi_{w C a}$.

(4) $\Phi_{c} \ll \Phi_{\delta_{0}}$.

(5) $\Phi_{c} \ll \Phi_{\text {lim }}$.

The first four can be found in [11]. For the last one, we observe the fact that each limited sequence is weakly Cauchy (see [1]) and (c).

Examples 4.9. The following operator ideals have desirable representations.

(1) The ideal $\mathfrak{U}$ of unconditionally summing operators

$$
\begin{aligned}
\mathfrak{U} & =\mathcal{O}\left(\mathcal{M}_{u c} / \mathcal{M}_{w u c}\right)=\mathcal{O}\left(\mathcal{M}_{u c}\right) \circ \mathcal{O}\left(\mathcal{M}_{w u c}\right)^{-1} \\
& =\mathcal{O}\left(\mathcal{M}_{c} / \mathcal{M}_{\delta_{0}}\right)=\mathcal{O}\left(\mathcal{M}_{c}\right) \circ \mathcal{O}\left(\mathcal{M}_{\delta_{0}}\right)^{-1}=\mathfrak{K} \circ \mathfrak{D}_{0}^{-1} .
\end{aligned}
$$

(2) The ideal $\mathfrak{V}$ of completely continuous operators

$$
\begin{aligned}
\mathfrak{V} & =\mathcal{O}\left(\Phi_{c} / \Phi_{w c}\right)=\mathcal{O}\left(\mathcal{M}_{c} / \mathcal{M}_{w c}\right)=\mathcal{O}\left(\mathcal{M}_{c}\right) \circ \mathcal{O}\left(\mathcal{M}_{w c}\right)^{-1}=\mathfrak{K} \circ \mathfrak{W}^{-1} \\
& =\mathcal{O}\left(\Phi_{c} / \Phi_{w C a}\right)=\mathcal{O}\left(\mathcal{M}_{c} / \mathcal{M}_{w C a}\right)=\mathcal{O}\left(\mathcal{M}_{c}\right) \circ \mathcal{O}\left(\mathcal{M}_{w C a}\right)^{-1}=\mathfrak{K} \circ \mathfrak{R}^{-1}
\end{aligned}
$$


(3) The ideal $w \mathfrak{S} \mathfrak{C}$ of weakly sequentially complete operators

$$
s \mathfrak{S C}=\mathcal{O}\left(\Phi_{w c} / \Phi_{w C a}\right)=\mathcal{O}\left(\mathcal{M}_{w c} / \mathcal{M}_{w C a}\right)=\mathcal{O}\left(\mathcal{M}_{w c}\right) \circ \mathcal{O}\left(\mathcal{M}_{w C a}\right)^{-1}=\mathfrak{W} \circ \mathfrak{R}^{-1} .
$$

(4) The ideal $\mathfrak{G P}$ of Gelfand-Phillips operators

$$
\mathfrak{G P}=\mathcal{O}\left(\Phi_{c} / \Phi_{\lim }\right)=\mathcal{O}\left(\mathcal{M}_{c} / \mathcal{M}_{\lim }\right)=\mathcal{O}\left(\mathcal{M}_{c}\right) \circ \mathcal{O}\left(\mathcal{M}_{\lim }\right)^{-1}=\mathfrak{K} \circ \mathfrak{L}_{\lim }^{-1}
$$

(5) The ideal $w \mathfrak{B S}$ of weakly Banach-Saks operators

$$
w \mathfrak{B S}=\mathcal{O}\left(\Phi_{\mathrm{BS}} / \Phi_{w c}\right)=\mathcal{O}\left(\mathcal{M}_{\mathrm{BS}} / \mathcal{M}_{w c}\right)=\mathcal{O}\left(\mathcal{M}_{\mathrm{BS}}\right) \circ \mathcal{O}\left(\mathcal{M}_{w c}\right)^{-1}=\mathfrak{B S} \circ \mathfrak{W}^{-1}
$$

See [11] for a proof of (a), (b) and (c). See [3] for (d). For (e), we simply recall that a $T$ in $\mathfrak{L}(E, F)$ is called weakly Banach-Saks if $T$ sends each weakly convergent sequence to a sequence possessing a Banach-Saks subsequence, by definition.

Proposition 4.10 ([1] ). Let $\mathfrak{A}$ be a surjective operator ideal such that $\mathfrak{A}$ is idempotent, i.e., $\mathfrak{A}^{2}=\mathfrak{A}$. Then for any operator ideal $\mathfrak{B} \supseteq \mathfrak{A}$, there exists an operator ideal $\mathfrak{C}$ such that

$$
\mathfrak{A}=\mathfrak{C} \circ \mathfrak{B}^{\text {sur }},
$$

and $\mathfrak{C}$ can be chosen to be $\mathfrak{A} \circ\left(\mathfrak{B}^{\text {sur }}\right)^{-1}$.

Proof. We present a proof here for completeness. Denote

$$
\mathcal{M}_{\mathfrak{A}}:=\mathcal{M}(\mathfrak{A}), \quad \mathcal{M}_{\mathfrak{B}}:=\mathcal{M}(\mathfrak{B})
$$

and set

$$
\mathfrak{C}:=\mathcal{O}\left(\mathcal{M}_{\mathfrak{A}} / \mathcal{M}_{\mathfrak{B}}\right)
$$

Now the facts $\mathfrak{A} \subseteq \mathfrak{B}$ and

$$
\mathfrak{A} \subseteq \mathfrak{C}=\mathcal{O}\left(\mathcal{M}_{\mathfrak{A}}\right) \circ \mathcal{O}\left(\mathcal{M}_{\mathfrak{B}}\right)^{-1}=\mathfrak{A} \circ\left(\mathfrak{B}^{\text {sur }}\right)^{-1}
$$

implies that

$$
\mathfrak{A}=\mathfrak{A}^{2} \subseteq \mathfrak{C} \circ \mathfrak{B} \subseteq \mathfrak{C} \circ \mathfrak{B}^{\text {sur }} .
$$

On the other hand, if $T \in \mathfrak{C}^{*} \mathfrak{B}^{\operatorname{sur}}(E, F)$ then $T=R S$ for some $R \in \mathfrak{C}(G, F)=$ $L^{\times}\left(G^{\mathcal{M}_{\mathfrak{B}}}, F^{\mathcal{M}_{\mathfrak{A}}}\right) \cap \mathfrak{L}(G, F)$ and $S \in \mathfrak{B}^{\text {sur }}(E, G)=L^{\times}\left(E, G^{\mathcal{M}_{\mathfrak{B}}}\right) \cap \mathfrak{L}(E, G)$ with some Banach space $G$. Hence,

$$
T \in L^{\times}\left(E, F^{\mathcal{M}_{\mathfrak{A}}}\right) \cap \mathfrak{L}(E, F)=\mathfrak{A}(E, F) .
$$


Examples 4.11 ([11]). (1) Since the surjective ideal $\mathfrak{K}$ of compact operators is idempotent and contained in the surjective ideal $\mathfrak{W}, \mathfrak{R}$, and $\mathfrak{D}_{0}$ of weakly compact operators, Rosenthal compact operators, and $\delta_{0}$-compact operators, respectively, we have

(a) $\mathfrak{K}=\mathfrak{V} \circ \mathfrak{W}$ since $\mathfrak{V}=\mathcal{O}\left(\mathcal{M}_{c} / \mathcal{M}_{w c}\right)=\mathfrak{K} \circ \mathfrak{W}^{-1}$;

(b) $\mathfrak{K}=\mathfrak{V} \circ \mathfrak{R}$ since $\mathfrak{V}=\mathcal{O}\left(\mathcal{M}_{c} / \mathcal{M}_{w c A}\right)=\mathfrak{K} \circ \mathfrak{R}^{-1}$;

(c) $\mathfrak{K}=\mathfrak{U} \circ \mathfrak{D}_{0}$ since $\mathfrak{U}=\mathcal{O}\left(\mathcal{M}_{c} / \mathcal{M}_{\delta_{0}}\right)=\mathfrak{K} \circ \mathfrak{D}_{0}{ }^{-1}$.

(2) Since the ideal $\mathfrak{W}$ of weakly compact operators is idempotent and contained in the surjective ideal $\mathfrak{R}$ of Rosenthal compact operators, we have

$$
\mathfrak{W}=w \mathfrak{S} \mathfrak{C} \circ \mathfrak{R}
$$

where $w \mathfrak{S} \mathfrak{C}=\mathcal{O}\left(\mathcal{M}_{w c} / \mathcal{M}_{w C a}\right)=\mathfrak{W} \circ \mathfrak{R}^{-1}$ is the ideal of weakly sequentially complete operators.

\section{REFERENCES}

[1] J. Bourgain and J. Diestel, "Limited operators and strictly cosingularity", Math. Nachr., 119 (1984), 55-58.

[2] J. Conradie and G. West, "Topological and bornological characterisations of ideals in von Neumann algebras: II", Integral Equations Operator Theory, 23 (1995), no. 1, 49-60.

[3] L. Drewnowski, "On Banach spaces with the Gelfand-Phillips property", Math. Z., 93 (1986), 405-411.

[4] H. Hogbe-Nlend, Bornologies and functional analysis, Math. Studies 26, North-Holland, Amsterdam, 1977.

[5] H. Hogbe-Nlend, Nuclear and co-nuclear spaces, Math. Studies 52, North-Holland, Amsterdam, 1981.

[6] A. Pietsch, Operator Ideals, North-Holland, Amsterdam, 1980.

[7] D. Randtke, "Characterizations of precompact maps, Schwartz spaces and nuclear spaces", Trans. Amer. Math. Soc., 165 (1972), 87-101.

[8] H. H. Schaefer, Topological Vector Spaces, Springer-Verlag, Berlin-Heidelberg-New York, 1971.

[9] I. Stephani, "Injektive operatorenideale über der gesamtheit aller Banachräume und ihre topologische erzeugung", Studia Math., 38 (1970), 105-124.

[10] I. Stephani, "Surjektive operatorenideale über der gesamtheit aller Banachräume und ihre Erzeugung", Beiträge Analysis, . 5 (1973), 75-89.

[11] I. Stephani, "Generating system of sets and quotients of surjective operator ideals", Math. Nachr., 99 (1980), 13-27.

[12] I. Stephani, "Generating topologies and quotients of injective operator ideals", in "Banach Space Theory and Its Application (proceedings, Bucharest 1981)", Lecture Notes in Math., 991, SpringerVerlag, Berlin-Heidelberg-New York, 1983, 239-255.

[13] G. West, "Topological and bornological characterisations of ideals in von Neumann algebras: I", Integral Equations Operator Theory, 22 (1995), no. 3, 352-359.

[14] N.-C. Wong, Operator ideals on locally convex spaces, Master Thesis, The Chinese University of Hong Kong, 1987.

[15] N.-C. Wong, "Topologies and bornologies determined by operator ideals, II", Studia Math. 111(2) (1994), 153-162. 
[16] N.-C. Wong, "The triangle of operators, topologies, bornologies", in Third International Congress of Chinese Mathematicians. Part 1, 2, 395-421, AMS/IP Stud. Adv. Math., 42, Part 1, 2, Amer. Math. Soc., Providence, RI, 2008. arXiv:math/0506183v1 [math.FA]

[17] N.-C. Wong and Y.-C. Wong, "The bornologically surjective hull of an operator ideal on locally convex spaces", Math. Narch., 160 (1993), 265-275.

[18] Y.-C. Wong and N.-C. Wong, "Topologies and bornologies determined by operator ideals", Math. Ann., 282 (1988), 587-614.

Department of Applied Mathematics, National Sun Yat-Sen University, and National Center for Theoretical Sciences, Kaohsiung, 80424, Taiwan, R.O.C.

E-mail address: wong@math.nsysu.edu.tw 